\title{
TWO PERIODIC SOLUTIONS OF NEUTRAL DIFFERENCE EQUATIONS MODELLING PHYSIOLOGICAL PROCESSES
}

\author{
JUN WU AND YICHENG LIU
}

Received 15 April 2006; Accepted 25 July 2006

We establish existence, multiplicity, and nonexistence of periodic solutions for a class of first-order neutral difference equations modelling physiological processes and conditions. Our approach is based on a fixed point theorem in cones as well as some analysis techniques.

Copyright (c) $2006 \mathrm{~J}$. Wu and Y. Liu. This is an open access article distributed under the Creative Commons Attribution License, which permits unrestricted use, distribution, and reproduction in any medium, provided the original work is properly cited.

\section{Introduction}

The existence of periodic solutions for difference equations has been extensively considered by many authors $[1,4,8,9,12,16]$. Recently, existence of multiple solutions of functional differential equations has been studied and some results have been obtained $[6,14,18]$. Wang [14] investigated existence, multiplicity, and nonexistence of positive periodic solutions for the equation

$$
\frac{d}{d t} x(t)=a(t) g(x(t)) x(t)-\lambda b(t) f(x(t-\tau(t)))
$$

where $\lambda$ is a positive parameter. Chow [2], Smith and Kuang [13], and many others studied the type of equations or their generalized forms. This type of equations has been proposed as models for a variety of physiological processes and conditions including production of blood cells, respiration, and cardiac arrhythmias [11, 15].

To our best knowledge, few papers are on multiplicity of periodic solutions of neutral functional difference systems. In this paper, we consider the following first-order neutral difference equation:

$$
\Delta(x(n)-c x(n-\delta))=a(n) g(x(n)) x(n)-\lambda b(n) f(x(n-\tau(n))), \quad n \in \mathbb{Z},
$$


2 Two solutions of neutral difference equations

where $\mathbb{Z}$ is the set of integers, $\Delta x(n)=x(n+1)-x(n), \lambda$ is a positive parameter, $c$ is a constant, and $|c| \neq 1, \delta$ is a positive integer, $a(n), b(n)$, and $\tau(n)$ are positive $T$-periodic sequences, $T \in \mathbb{N}$.

Let $N^{*}=\{0,1,2, \ldots, T-1\}$ and

$$
\begin{gathered}
f_{0}=\lim _{u \rightarrow 0^{+}} \frac{f(u)}{u}, \quad f_{\infty}=\lim _{u \rightarrow \infty} \frac{f(u)}{u}, \\
i_{0}=\text { number of zeros in the set }\left\{f_{0}, f_{\infty}\right\}, \\
i_{\infty}=\text { number of infinities in the set }\left\{f_{0}, f_{\infty}\right\} .
\end{gathered}
$$

It is clear that $i_{0}, i_{\infty}=0,1$, or 2 . Then we should show that (1.2) has $i_{0}$ or $i_{\infty}$ periodic solution(s) for some certain $\lambda$, respectively. In what follows, we set

$$
X=\{x \mid x(n), x(n+T) \equiv x(n), n \in \mathbb{Z}\}
$$

with the norm defined by $\|x\|_{X}=\max \left\{|x(n)|: n \in N^{*}\right\}$. Then $X$ is a Banach space. Let $A: X \rightarrow X$ be defined by $(A x)(n)=x(n)-c x(n-\delta)$.

Lemma 1.1. If $|c| \neq 1$, then $A$ has continuous bounded inverse $A^{-1}$ on $X$ and for all $x \in X$,

$$
\begin{aligned}
\left(A^{-1} x\right)(n)= & \begin{cases}\sum_{j \geq 0} c^{j} x(n-j \delta), & \text { if }|c|<1, \\
-\sum_{j \geq 1} c^{-j} x(n+j \delta), & \text { if }|c|>1, n \in \mathbb{Z},\end{cases} \\
& \left\|A^{-1} x\right\|_{X} \leq \frac{\|x\|_{X}}{|1-| c||} .
\end{aligned}
$$

Proof. According to $[10,17]$, we can get equality (1.5) and then verify the results of Lemma 1.1.

We consider the following assumptions.

$\left(\mathrm{E}_{1}\right) a(n), b(n)$ are positive $T$-periodic sequences, $\tau(n)$ is a positive $T$-periodic integer sequence.

$\left(\mathrm{E}_{2}\right) f, g \in \mathbb{C}([0, \infty),[0, \infty))$ and there exist two positive constants $l, L$ such that $0<l \leq$ $g(u) \leq L<+\infty$ for $u \in \mathbb{R} ; f(u)>0$ for $u>0$.

Define

$$
A_{1}=\frac{1}{\prod_{r=n}^{n+T-1}[a(r) L+1]-1}, \quad B=\frac{\prod_{r=n}^{n+T-1}[a(r) L+1]}{\prod_{r=n}^{n+T-1}[a(r) l+1]-1},
$$


and $\alpha=A_{1} / B$, for any $r>0$, we denote

$$
\begin{gathered}
M(r)=\max \left\{f(t): 0 \leq t \leq \frac{r}{1-|c|}\right\}, \\
m(r)=\min \left\{f(t): \frac{\alpha-|c|}{1-c^{2}} r \leq t \leq \frac{r}{1-|c|}\right\}, \\
k=\min \left\{\alpha, \frac{1}{1+B L \Sigma_{s=0}^{T-1} a(s)}\right\} .
\end{gathered}
$$

We aim to establish existence, multiplicity, and nonexistence of positive $T$-periodic solutions for first-order neutral difference equation (1.2). Our approach is based on a fixed point theorem in cones as well as some analysis techniques which are used by Wang [14]. The rest of this paper is organized as follows. Section 2 is about statement of the method (a fixed point theorem in cones) and some lemmas which play important roles in proofs of main results; in Section 3, we establish our main results and give an example to illustrate the applicability of our results.

\section{Preliminaries}

We first state the following well-known result. For the proof, we refer to the classical works $[3,5,7]$.

Lemma 2.1 (Deimling [3], Guo and Lakshmikantham [5], and Krasnosel'skiu [7]). Let $E$ be a Banach space and $K$ a cone in $E$. For $r>0$, define $K_{r}=\{u \in K:\|u\|<r\}$. Assume that $T: \bar{K}_{r} \rightarrow K$ is completely continuous such that $T x \neq x$ for $x \in \partial K_{r}=\{u \in K:\|u\|=r\}$.

(i) If $\|T x\| \geq\|x\|$ for any $x \in \partial K_{r}$, then $i\left(T, K_{r}, K\right)=0$.

(ii) If $\|T x\| \leq\|x\|$ for any $x \in \partial K_{r}$, then $i\left(T, K_{r}, K\right)=1$.

Next, we transfer existence of positive $T$-periodic solutions of (1.2) into existence of positive fixed points of some fixed point mapping.

In order to establish existence, multiplicity, and nonexistence of positive $T$-periodic solutions for (1.2), we first consider the following equation:

$$
\Delta y(n)=a(n) g\left(\left(A^{-1} y\right)(n)\right)\left(A^{-1} y\right)(n)-\lambda b(n) f\left(\left(A^{-1} y\right)(n-\tau(n))\right),
$$

where $A^{-1}$ is defined by (1.5). By Lemma 1.1 and the definition of $A$ and $A^{-1}$, we conclude the following.

Lemma 2.2. $y(n)$ is a T-periodic solution of (2.1) if and only if $\left(A^{-1} y\right)(n)$ is a T-periodic solution of (1.2).

Aiming to apply Lemma 2.1 to (2.1), we rewrite (2.1) as

$$
\Delta y(n)=a(n) g\left(\left(A^{-1} y\right)(n)\right) y(n)-\left[a(n) G(y(n))+\lambda b(n) f\left(\left(A^{-1} y\right)(n-\tau(n))\right)\right]
$$


4 Two solutions of neutral difference equations

where

$$
G(y(n))=-c g\left(\left(A^{-1} y\right)(n)\right)\left(A^{-1} y\right)(n-\tau) .
$$

A cone $K$ in $X$ is defined by

$$
K=\left\{u \in X: u(n) \geq \alpha\|u\|_{X}, n \in \mathbb{Z}\right\} .
$$

For $r>0$, define $\Omega_{r}$ by $\Omega_{r}=\left\{u \in K:\|u\|_{X}<r\right\}$ and $\partial \Omega_{r}=\left\{u \in K:\|u\|_{X}=r\right\}$. Let the operator $Q: K \rightarrow X$ be defined by

$$
Q u(n)=\sum_{s=n}^{n+T-1} K_{u}(n, s)\left[a(s) G(u(s))+\lambda b(s) f\left(\left(A^{-1} u\right)(s-\tau(s))\right)\right], \quad n \in \mathbb{Z},
$$

where

$$
K_{u}(n, s)=\frac{\prod_{r=s+1}^{n+T-1}\left[a(r) g\left(\left(A^{-1} u\right)(r)\right)+1\right]}{\prod_{r=n}^{n+T-1}\left[a(r) g\left(\left(A^{-1} u\right)(r)\right)+1\right]-1}, \quad n, s \in \mathbb{Z}, n \leq s \leq n+T-1 .
$$

Assumption $\left(\mathrm{E}_{2}\right)$ implies that

$$
0<A_{1} \leq K_{u}(n, s) \leq B, \quad n, s \in \mathbb{Z}, n \leq s \leq n+T-1 .
$$

Lemma 2.3. The positive T-periodic solution of (2.1) is equivalent to the fixed point of $Q$ in $K$.

Lemma 2.4. If assumptions $\left(E_{1}\right)$ and $\left(E_{2}\right)$ hold, $c \in(-\alpha, 0]$, and $y \in K$, then

(a) $\left((\alpha-|c|) /\left(1-c^{2}\right)\right)\|y\|_{X} \leq\left(A^{-1} y\right)(n) \leq(1 /(1-|c|))\|y\|_{X}$,

(b) $l|c|\left((\alpha-|c|) /\left(1-c^{2}\right)\right)\|y\|_{X} \leq G(y(n)) \leq(L|c| /(1-|c|))\|y\|_{X}, n \in N^{*}$.

Proof

Part (a). Since $-\alpha<c \leq 0$, it follows from Lemma 1.1 that

$$
\begin{aligned}
\left(A^{-1} y\right)(n)= & \sum_{j \geq 0} c^{j} y(n-j \delta) \\
= & \sum_{j \geq 0} c^{2 j} y(n-2 j \delta)-\sum_{j \geq 1}|c|^{2 j-1} y(n-(2 j-1) \delta) \\
\geq & \frac{\alpha-|c|}{1-c^{2}}\|y\|_{X}, \quad n \in N^{*} \\
& \quad\left(A^{-1} y\right)(n) \leq \frac{1}{1-|c|}\|y\|_{X}
\end{aligned}
$$

Part (b). From part (a) and the assumption $\left(\mathrm{E}_{2}\right)$, for any $n \in \mathbb{Z}$, we get

$$
l|c| \frac{\alpha-|c|}{1-c^{2}}\|y\|_{X} \leq G(y(n)) \leq \frac{L|c|}{1-|c|}\|y\|_{X}
$$

Lemma 2.5. If assumptions $\left(E_{1}\right)$ and $\left(E_{2}\right)$ hold and $c \in(-\alpha, 0]$, then $Q(K) \subset K$ and $Q$ : $K \rightarrow K$ is completely continuous. 
Proof. By Lemma 1.1, similar to the proof of Lemma 2.2 in [7], we can prove Lemma 2.5.

Lemma 2.6. If assumptions $\left(E_{1}\right)$ and $\left(E_{2}\right)$ hold and $c \in(-\alpha, 0]$, then $y(n)$ is the fixed point of $Q$ in $K$ if and only if $\left(A^{-1} y\right)(n)$ is a positive T-periodic solution of (1.2).

Proof. If $y(n)$ is the fixed point of $Q$ in $K, y(n)$ is a positive T-periodic solution of (2.1) and $y \in K$ by Lemma 2.3. It follows from Lemmas 2.2 and 2.4 that $\left(A^{-1} y\right)(n)$ is a $T$-periodic solution of $(1.2)$ and $\left(A^{-1} y\right)(n) \geq\left((\alpha-|c|) /\left(1-c^{2}\right)\right)\|y\|_{X}>0$. Therefore, $\left(A^{-1} y\right)(n)$ is a positive $T$-periodic solution of (1.2).

If there exists $y(n)$ such that $\left(A^{-1} y\right)(n)$ is a positive $T$-periodic solution of (1.2), then $y(n)$ is a $T$-periodic solution of (2.1) by Lemma 2.2. From the definition of $A^{-1}$ and $c \in(-\alpha, 0], y(n)=\left(A^{-1} y\right)(n)-c\left(A^{-1} y\right)(n-\delta)>0$. Lemmas 2.3 and 2.5 imply that $y(n)$ is the fixed point of $Q$ in $K$.

Lemma 2.7. Assumptions $\left(E_{1}\right)$ and $\left(E_{2}\right)$ hold and $c \in(-\alpha, 0], \eta>0$. If $f\left(\left(A^{-1} y\right)(n-\right.$ $\tau(n))) \geq\left(A^{-1} y\right)(n-\tau(n)) \eta$ for any $y \in K$ and $n \in \mathbb{Z}$, then

$$
\|Q y\|_{X} \geq \lambda A_{1} \eta \sum_{s=0}^{T-1} b(s) \frac{\alpha-|c|}{1-c^{2}}\|y\|_{X} .
$$

Proof. By Lemma 2.4, for any $y \in K$ and $n \in \mathbb{Z}, G(y(n)) \geq 0$ as $c \in(-\alpha, 0]$. Therefore,

$$
\begin{aligned}
Q y(n) & \geq \lambda A_{1} \sum_{s=n}^{n+T-1} b(s) f\left(\left(A^{-1} y\right)(s-\tau(s))\right)=\lambda A_{1} \sum_{s=0}^{T-1} b(s) f\left(\left(A^{-1} y\right)(s-\tau(s))\right) \\
& \geq \lambda A_{1} \eta \sum_{s=0}^{T-1} b(s)\left(A^{-1} y\right)(s-\tau(s)) \geq \lambda A_{1} \eta \sum_{s=0}^{T-1} b(s) \frac{\alpha-|c|}{1-c^{2}}\|y\|_{X} .
\end{aligned}
$$

That is,

$$
\|Q y\|_{X} \geq \lambda A_{1} \eta \Sigma_{s=0}^{T-1} b(s) \frac{\alpha-|c|}{1-c^{2}}\|y\|_{X}
$$

Lemma 2.8. Assumptions $\left(E_{1}\right)$ and $\left(E_{2}\right)$ hold and $c \in(-\alpha, 0]$. For any $n \in \mathbb{Z}$, if there exists $\varepsilon>0$ such that $f\left(\left(A^{-1} y\right)(n-\tau(n))\right) \leq\left(A^{-1} y\right)(n-\tau(n)) \mathcal{E}$, then

$$
\|Q y\|_{X} \leq \frac{B \sum_{s=0}^{T-1}[L|c| a(s)+\lambda \varepsilon b(s)]}{1-|c|}\|y\|_{X} .
$$

Proof. From Lemmas 1.1 and 2.4, we have

$$
\begin{aligned}
\|Q y\|_{X} & \leq B \sum_{s=0}^{T-1}\left[a(s) G(y(s))+\lambda b(s) f\left(\left(A^{-1} y\right)(s-\tau(s))\right)\right] \\
& \leq B \Sigma_{s=0}^{T-1}\left[a(s) \frac{L|c|}{1-|c|}\|y\|_{X}+\lambda b(s) \varepsilon\left(A^{-1} y\right)(s-\tau(s))\right] \\
& \leq \frac{B \Sigma_{s=0}^{T-1}[L|c| a(s)+\lambda \varepsilon b(s)]}{1-|c|}\|y\|_{X} .
\end{aligned}
$$


Lemma 2.9. Assumptions $\left(E_{1}\right)$ and $\left(E_{2}\right)$ hold and $c \in(-\alpha, 0]$. For $y \in \partial \Omega_{r}, r>0$, one can obtain

$$
\|Q y\|_{X} \geq \lambda A_{1} m(r) \Sigma_{s=0}^{T-1} b(s) .
$$

Proof. Since $y \in \partial \Omega_{r}$, by Lemma 2.4, $\left((\alpha-|c|) /\left(1-c^{2}\right)\right) r \leq\left(A^{-1} y\right)(n-\tau(n)) \leq r /(1-$ $|c|)$. So $f\left(\left(A^{-1} y\right)(n-\tau(n))\right) \geq m(r)$ for $y \in \partial \Omega_{r}$ and $n \in \mathbb{Z}$. Similar to the proof of Lemma 2.7, we can obtain Lemma 2.9.

Lemma 2.10. Assumptions $\left(E_{1}\right)$ and $\left(E_{2}\right)$ hold and $c \in(-\alpha, 0]$. If $y \in \partial \Omega_{r}, r>0$, then

$$
\|Q y\|_{X} \leq B \Sigma_{s=0}^{T-1}\left[\lambda b(s) M(r)+\frac{L|c| a(s) r}{1-|c|}\right] .
$$

Proof. By $y \in \partial \Omega_{r}$ and Lemma 1.1, $0 \leq\left(A^{-1} y\right)(n-\tau(n)) \leq r /(1-|c|)$. So $f\left(\left(A^{-1} y\right)(n-\right.$ $\tau(n))) \leq M(r)$ for any $y \in \partial \Omega_{r}$ and $n \in \mathbb{Z}$. From The proof of Lemma 2.8, we can similarly prove Lemma 2.10 .

\section{Main results}

We state our main results as follows.

Theorem 3.1. Suppose that assumptions $\left(E_{1}\right),\left(E_{2}\right)$ hold and $-k<c \leq 0$.

(a) If $i_{0}=1$ or 2 , then (1.2) has $i_{0}$ positive $T$-periodic solution(s) for $\lambda>1 / A_{1} m(1) \sum_{s=0}^{T-1} b(s)$ $>0$.

(b) If $i_{\infty}=1$ or 2 , then (1.2) has $i_{\infty}$ positive T-periodic solution(s) for $0<\lambda<(1-|c|-$ $\left.B L|c| \sum_{s=0}^{T-1} a(s)\right) / B M(1) \sum_{s=0}^{T-1} b(s)(1-|c|)$.

(c) If $i_{\infty}=0$ or $i_{0}=0$, then (1.2) has no positive T-periodic solution for sufficiently small or large $\lambda>0$, respectively.

Theorem 3.2. Suppose that assumptions $\left(E_{1}\right),\left(E_{2}\right)$ hold and $-k<c \leq 0$.

(a) If there exists a constant $c_{1}>0$ such that $f(u) \geq c_{1} u$ for $u \in[0,+\infty)$, then (1.2) has no positive $T$-periodic solution for $\lambda>\left(1-c^{2}\right) / A_{1} c_{1}(\alpha-|c|) \sum_{s=0}^{T-1} b(s)$.

(b) If there exists a constant $c_{2}>0$ such that $f(u) \leq c_{2} u$ for $u \in[0,+\infty)$, then (1.2) has no positive $T$-periodic solution for $0<\lambda<\left(1-|c|-B L|c| \sum_{s=0}^{T-1} a(s)\right) / B c_{2} \sum_{s=0}^{T-1} b(s)$.

Theorem 3.3. Suppose that assumptions $\left(E_{1}\right),\left(E_{2}\right)$ hold and $-k<c \leq 0$. If $i_{0}=i_{\infty}=0$ and

$$
\frac{1-c^{2}}{\max \left\{f_{\infty}, f_{0}\right\} A_{1}(\alpha-|c|) \sum_{s=0}^{T-1} b(s)}<\lambda<\frac{1-|c|-B L|c| \sum_{s=0}^{T-1} a(s)}{\min \left\{f_{0}, f_{\infty}\right\} B \sum_{s=0}^{T-1} b(s)},
$$

then (1.2) has one positive T-periodic solution.

Proof of Theorem 3.1

Part (a). Take $r_{1}=1$ and $\lambda_{0}=1 / A_{1} m\left(r_{1}\right) \sum_{s=0}^{T-1} b(s)>0$. For any $y \in \partial \Omega_{r_{1}}$ and $\lambda>\lambda_{0}$, it follows from Lemma 2.9 that

$$
\|Q y\|_{X}>\|y\|_{X}, \quad y \in \partial \Omega_{r_{1}} .
$$

From Lemma 2.1, $i\left(Q, \Omega_{r_{1}}, K\right)=0$. 
Case 1. If $f_{0}=0$, then for any $\varepsilon>0$, we can choose $0<\bar{r}_{2}<r_{1}$ such that $f(u) \leq \varepsilon u$ for $0 \leq u \leq \bar{r}_{2}$. Since $-k<c \leq 0,1>B L|c| \sum_{s=0}^{T-1} a(s) /(1-|c|)$. Take $\varepsilon>0$ satisfying

$$
\frac{\lambda B \varepsilon \sum_{s=0}^{T-1} b(s)}{1-|c|}<1-\frac{B L|c| \sum_{s=0}^{T-1} a(s)}{1-|c|}
$$

Let $r_{2}=(1-|c|) \bar{r}_{2}$. If $y \in \partial \Omega_{r_{2}}$, then $0 \leq\left(A^{-1} y\right)(n-\tau(n)) \leq 1 /(1-|c|)\|y\|_{X} \leq \bar{r}_{2}$. So $f\left(\left(A^{-1} y\right)(n-\tau(n))\right) \leq \varepsilon\left(A^{-1} y\right)(n-\tau(n))$ for any $y \in \partial \Omega_{r_{2}}$ and $n \in \mathbb{Z}$. By Lemma 2.8 and inequality (3.3), for all $y \in \partial \Omega_{r_{2}}$, we have

$$
\|Q y\|_{X} \leq \frac{\lambda B \varepsilon \sum_{s=0}^{T-1} b(s)+B L|c| \sum_{s=0}^{T-1} a(s)}{1-|c|}\|y\|_{X}<\|y\|_{X} .
$$

Lemma 2.1 implies that $i\left(Q, \Omega_{r_{2}}, K\right)=1$. Thus $i\left(Q, \Omega_{r_{1}} \backslash \bar{\Omega}_{r_{2}}, K\right)=-1$ and $Q$ has a fixed point $y(n)$ in $\Omega_{r_{1}} \backslash \bar{\Omega}_{r_{2}}$. It follows from Lemma 2.6 that (1.2) has at least one positive $T$-periodic solution $\left(A^{-1} y\right)(n)$ for $\lambda>\lambda_{0}$.

Case 2. If $f_{\infty}=0$, then there exists a constant $\tilde{H}>0$ for any $\varepsilon>0$ such that $f(u) \leq \varepsilon u$ for all $u \geq \tilde{H} .-k<c \leq 0$ shows that $1>B L|c| \sum_{s=0}^{T-1} a(s) /(1-|c|)$. So we can choose $\varepsilon>0$ satisfying inequality (3.3).

Take $r_{3}=\max \left\{2 r_{1},\left(\left(1-c^{2}\right) /(\alpha-|c|)\right) \tilde{H}\right\}$. For any $y \in \partial \Omega_{r_{3}}$, since $\left(A^{-1} y\right)(n-\tau(n)) \geq$ $\left((\alpha-|c|) /\left(1-c^{2}\right)\right)\|y\|_{X} \geq \tilde{H}, f\left(\left(A^{-1} y\right)(n-\tau(n))\right) \leq \varepsilon\left(A^{-1} y\right)(n-\tau(n))$. From Lemma 2.8 and inequality (3.3), for each $y \in \partial \Omega_{r_{3}}$, we get

$$
\|Q y\|_{X} \leq \frac{\lambda B \varepsilon \sum_{s=0}^{T-1} b(s)+B L|c| \sum_{s=0}^{T-1} a(s)}{1-|c|}\|y\|_{X}<\|y\|_{X} .
$$

It follows from Lemma 2.1 that $i\left(Q, \Omega_{r_{3}}, K\right)=1$. Therefore, $i\left(Q, \Omega_{r_{3}} \backslash \bar{\Omega}_{r_{1}}, K\right)=1$ and $Q$ has at least one fixed point $y(n)$ in $\Omega_{r_{3}} \backslash \bar{\Omega}_{r_{1}}$. By Lemma 2.6, we conclude that (1.2) has at least one positive $T$-periodic solution $\left(A^{-1} y\right)(n)$ for $\lambda>\lambda_{0}$.

Case 3. If $f_{\infty}=f_{0}=0$, from the above arguments, there exist $r_{1}, r_{2}$, and $r_{3}$ with $0<r_{2}<$ $r_{1}<r_{3}$ such that $Q$ has fixed points $y_{1}(n)$ and $y_{2}(n)$ in $\Omega_{r_{1}} \backslash \bar{\Omega}_{r_{2}}$ and $\Omega_{r_{3}} \backslash \bar{\Omega}_{r_{1}}$, respectively. By Lemma 2.6, for any $\lambda>\lambda_{0}$, (1.2) has at least two positive $T$-periodic solutions $\left(A^{-1} y_{1}\right)(n)$ and $\left(A^{-1} y_{2}\right)(n)$.

Part (b). $\quad-k<c \leq 0$ implies that $1>B L|c| \sum_{s=0}^{T-1} a(s) /(1-|c|)$. Let $r_{1}=1$ and $\lambda_{1}=(1-$ $\left.|c|-B L|c| \sum_{s=0}^{T-1} a(s)\right) / B M\left(r_{1}\right) \sum_{s=0}^{T-1} b(s)(1-|c|)>0$. From Lemma 2.10, for any $y \in \partial \Omega_{r_{1}}$ and $0<\lambda<\lambda_{1}$, we have

$$
\|Q y\|_{X}<\|y\|_{X}
$$

By Lemma 2.1, $i\left(Q, \Omega_{r_{1}}, K\right)=1$.

Case 1. If $f_{0}=\infty$, then for any $\eta>0$, there exists $0<\bar{r}_{2}<r_{1}$ such that $f(u) \geq \eta u$ for each $0 \leq u \leq \bar{r}_{2}$. Take $\eta>0$ satisfying

$$
\lambda A_{1} \eta \frac{\alpha-|c|}{1-c^{2}} \sum_{s=0}^{T-1} b(s)>1 .
$$


Let $r_{2}=(1-|c|) \bar{r}_{2}$. For any $y \in \partial \Omega_{r_{2}}, 0 \leq\left(A^{-1} y\right)(n-\tau(n)) \leq(1 /(1-|c|))\|y\|_{X} \leq \bar{r}_{2}$. Thus $f\left(\left(A^{-1} y\right)(n-\tau(n))\right) \geq \eta\left(A^{-1} y\right)(n-\tau(n))$ for $y \in \partial \Omega_{r_{2}}$ and $n \in \mathbb{Z}$. By Lemma 2.7 and inequality (3.7), for any $y \in \partial \Omega_{r_{2}}$, we get

$$
\|Q y\|_{X} \geq \lambda A_{1} \eta \frac{\alpha-|c|}{1-c^{2}} \sum_{s=0}^{T-1} b(s)\|y\|_{X}>\|y\|_{X} .
$$

Lemma 2.1 tells that $i\left(Q, \Omega_{r_{2}}, K\right)=0$. So $i\left(Q, \Omega_{r_{1}} \backslash \bar{\Omega}_{r_{2}}, K\right)=1$ and $Q$ has at least one fixed point $y(n)$ in $\Omega_{r_{1}} \backslash \bar{\Omega}_{r_{2}}$. From Lemma 2.6, $\left(A^{-1} y\right)(n)$ is a positive $T$-periodic solution of (1.2) for $\lambda \in\left(0, \lambda_{1}\right)$.

Case 2. If $f_{\infty}=\infty$, then for any $\eta>0$, we can find $\tilde{H}>0$ satisfying that $f(u) \geq \eta u$ for each $u \geq \tilde{H}$. Take $\eta>0$ such that inequality (3.7) holds.

Let $r_{3}=\max \left\{2 r_{1},\left(\left(1-c^{2}\right) /(\alpha-|c|)\right) \tilde{H}\right\}$. As $y \in \partial \Omega_{r_{3}},\left(A^{-1} y\right)(n-\tau(n)) \geq((\alpha-|c|) /(1-$ $\left.\left.c^{2}\right)\right)\|y\|_{X} \geq \tilde{H}$. Then $f\left(\left(A^{-1} y\right)(n-\tau(n))\right) \geq \eta\left(A^{-1} y\right)(n-\tau(n))$ for any $y \in \partial \Omega_{r_{3}}$. For any $y \in \partial \Omega_{r_{3}}$, it follows from Lemma 2.7 and inequality (3.7) that

$$
\|Q y\|_{X} \geq \lambda A_{1} \eta \frac{\alpha-|c|}{1-c^{2}} \sum_{s=0}^{T-1} b(s)\|y\|_{X}>\|y\|_{X}
$$

By Lemma 2.1, we obtain $i\left(Q, \Omega_{r_{3}}, K\right)=0$. Thus, $i\left(Q, \Omega_{r_{3}} \backslash \bar{\Omega}_{r_{1}}, K\right)=-1$ and $Q$ has at least one fixed point $y(n)$ in $\Omega_{r_{3}} \backslash \bar{\Omega}_{r_{1}}$. Lemma 2.6 shows that $\left(A^{-1} y\right)(n)$ is a positive $T$-periodic solution of (1.2) for $\lambda \in\left(0, \lambda_{1}\right)$.

Case 3. If $f_{\infty}=f_{0}=\infty$, from the arguments of Cases 1 and 2 in Part (b), there exist constants $0<r_{2}<r_{1}<r_{3}$ such that $Q$ has one fixed point in $\Omega_{r_{1}} \backslash \bar{\Omega}_{r_{2}}$ and $\Omega_{r_{3}} \backslash \bar{\Omega}_{r_{1}}$, respectively, denoting $y_{1}(n)$ and $y_{2}(n)$. That is, for any $\lambda \in\left(0, \lambda_{1}\right),(1.2)$ has at least two positive $T$-periodic solutions $\left(A^{-1} y_{1}\right)(n)$ and $\left(A^{-1} y_{2}\right)(n)$.

Part (c)

Case 1. If $i_{0}=0$, then $f_{0}>0$ and $f_{\infty}>0$. Letting $c_{1}=\min \{(f(u) / u): u>0\}>0$, we have

$$
f(u) \geq c_{1} u, \quad u \in[0,+\infty) .
$$

Take $\lambda_{2}=\left(1-c^{2}\right) /\left(A_{1} c_{1}(\alpha-|c|) \sum_{s=0}^{T-1} b(s)\right)$ and suppose that $u(n)$ is the positive $T$ periodic solution of (1.2) for $\lambda>\lambda_{2}$. For any $n \in \mathbb{Z}, f\left(A^{-1} u(n-\tau(n))\right) \geq c_{1} A^{-1} u(n-$ $\tau(n)) \geq\left(c_{1}(\alpha-|c|) /\left(1-c^{2}\right)\right)\|u\|_{X}$ and $Q u(n)=u(n)$. From Lemma 2.7, for $\lambda>\lambda_{2}$, we obtain

$$
\|u\|_{X}=\|Q u\|_{X} \geq \lambda A_{1} c_{1} \frac{\alpha-|c|}{1-c^{2}} \sum_{s=0}^{T-1} b(s)\|u\|_{X}>\|u\|_{X}
$$

which is a contradiction. Thus, when $i_{0}=0$ and $\lambda>\lambda_{2},(1.2)$ has no positive $T$-periodic solution.

Case 2. $i_{\infty}=0$ implies that $f_{0}<\infty f_{\infty}<\infty$. Since $-k<c \leq 0,1-|c|>B L|c| \sum_{s=0}^{T-1} a(s)$. Letting $c_{2}=\max \{f(u) / u: u>0\}>0$, we get

$$
f(u) \leq c_{2} u, \quad u \in[0,+\infty) .
$$

Take $\lambda_{3}=\left(1-|c|-B L|c| \sum_{s=0}^{T-1} a(s)\right) / B c_{2} \sum_{s=0}^{T-1} b(s)$. Suppose that $u(n)$ is the positive $T$-periodic solution of (1.2) corresponding to $\lambda \in\left(0, \lambda_{3}\right)$. For any $n \in \mathbb{Z}, f\left(A^{-1} u(n-\right.$ 
$\tau(n))) \leq c_{2} A^{-1} u(n-\tau(n)) \leq\left(c_{2} /(1-|c|)\right)\|u\|_{X}$ and $Q u(n)=u(n)$. Therefore, by Lemma 2.8 , for $\lambda \in\left(0, \lambda_{3}\right)$, we have

$$
\|u\|_{X}=\|Q u\|_{X} \leq \frac{\lambda B c_{2} \sum_{s=0}^{T-1} b(s)+B L|c| \sum_{s=0}^{T-1} a(s)}{1-|c|}\|u\|_{X}<\|u\|_{X},
$$

which is a contradiction. So, When $i_{\infty}=0,(1.2)$ has no positive $T$-periodic solution for any $0<\lambda<\lambda_{3}$.

Proof of Theorem 3.2. Following the proof of part (c) of Theorem 3.1, we can obtain this result immediately.

Proof of Theorem 3.3

Case 1. If $f_{0} \leq f_{\infty}$, then

$$
\frac{1-c^{2}}{f_{\infty} A_{1}(\alpha-|c|) \sum_{s=0}^{T-1} b(s)}<\lambda<\frac{1-|c|-B L|c| \sum_{s=0}^{T-1} a(s)}{f_{0} B \sum_{s=0}^{T-1} b(s)} .
$$

We can choose $0<\varepsilon<f_{\infty}$ such that

$$
\frac{1-c^{2}}{\left(f_{\infty}-\varepsilon\right) A_{1}(\alpha-|c|) \sum_{s=0}^{T-1} b(s)}<\lambda<\frac{1-|c|-B L|c| \sum_{s=0}^{T-1} a(s)}{\left(f_{0}+\varepsilon\right) B \sum_{s=0}^{T-1} b(s)} .
$$

From the definition of $f_{0}$, there exists $\bar{r}_{1}>0$ such that $f(u) \leq\left(f_{0}+\varepsilon\right) u$ for any $0 \leq u \leq$ $\bar{r}_{1}$. Take $r_{1}=(1-|c|) \bar{r}_{1}$. For $y \in \partial \Omega_{r_{1}}$, since $0 \leq\left(A^{-1} y\right)(n-\tau(n)) \leq(1 /(1-|c|))\|y\|_{X} \leq$ $\bar{r}_{1}$, then $f\left(\left(A^{-1} y\right)(n-\tau(n))\right) \leq\left(f_{0}+\varepsilon\right)\left(A^{-1} y\right)(n-\tau(n))$. By Lemma 2.8, for any $y \in$ $\partial \Omega_{r_{1}}$, we get

$$
\|Q y\|_{X} \leq \frac{B \lambda\left(f_{0}+\varepsilon\right) \sum_{s=0}^{T-1} b(s)+B L|c| \sum_{s=0}^{T-1} a(s)}{1-|c|}\|y\|_{X}<\|y\|_{X} .
$$

On the other hand, we can choose $\tilde{H}>0$ such that $f(u) \geq\left(f_{\infty}-\varepsilon\right) u$ for $u \geq \tilde{H}$. Let $r_{2}=\max \left\{2 r_{1},\left(\left(1-c^{2}\right) /(\alpha-|c|)\right) \tilde{H}\right\}$. If $y \in \partial \Omega_{r_{2}}$, then $\left(A^{-1} y\right)(n-\tau(n)) \geq((\alpha-|c|) /(1-$ $\left.\left.c^{2}\right)\right)\|y\|_{X} \geq \tilde{H}$. So $f\left(\left(A^{-1} y\right)(n-\tau(n))\right) \geq\left(f_{\infty}-\varepsilon\right)\left(A^{-1} y\right)(n-\tau(n))$ for any $y \in \partial \Omega_{r_{2}}$. From Lemma 2.7, for $y \in \partial \Omega_{r_{2}}$, we have

$$
\|Q y\|_{X} \geq \lambda\left(f_{\infty}-\varepsilon\right) A_{1} \frac{\alpha-|c|}{1-c^{2}} \sum_{s=0}^{T-1} b(s)\|y\|_{X}>\|y\|_{X} .
$$

It follows from Lemma 2.1 that

$$
i\left(Q, \Omega_{r_{1}}, K\right)=1, \quad i\left(Q, \Omega_{r_{2}}, K\right)=0, \quad i\left(Q, \Omega_{r_{2}} \backslash \bar{\Omega}_{r_{1}}, K\right)=-1 .
$$

Then $Q$ has at least one fixed point $y(n)$ in $\Omega_{r_{2}} \backslash \bar{\Omega}_{r_{1}}$. By Lemma 2.6, $\left(A^{-1} y\right)(n)$ is the positive $T$-periodic solution of (1.2).

Case 2. If $f_{0}>f_{\infty}$, then

$$
\frac{1-c^{2}}{f_{0} A_{1}(\alpha-|c|) \sum_{s=0}^{T-1} b(s)}<\lambda<\frac{1-|c|-B L|c| \sum_{s=0}^{T-1} a(s)}{f_{\infty} B \sum_{s=0}^{T-1} b(s)} .
$$


So we can take a constant $0<\varepsilon<f_{0}$ satisfying

$$
\frac{1-c^{2}}{\left(f_{0}-\varepsilon\right) A_{1}(\alpha-|c|) \sum_{s=0}^{T-1} b(s)}<\lambda<\frac{1-|c|-B L|c| \sum_{s=0}^{T-1} a(s)}{\left(f_{\infty}+\varepsilon\right) B \sum_{s=0}^{T-1} b(s)} .
$$

$0<f_{0}<\infty$ implies that there exists $\bar{r}_{1}>0$ such that for any $0 \leq u \leq \bar{r}_{1}, f(u) \geq\left(f_{0}-\varepsilon\right) u$.

Let $r_{1}=(1-|c|) \bar{r}_{1}$. If $y \in \partial \Omega_{r_{1}}$, then $0 \leq\left(A^{-1} y\right)(n-\tau(n)) \leq(1 /(1-|c|))\|y\|_{X} \leq \bar{r}_{1}$. So we have $f\left(\left(A^{-1} y\right)(n-\tau(n))\right) \geq\left(f_{0}-\varepsilon\right)\left(A^{-1} y\right)(n-\tau(n))$ for $y \in \partial \Omega_{r_{1}}$. From Lemma 2.7, for any $y \in \partial \Omega_{r_{1}}$, we obtain

$$
\|Q y\|_{X} \geq \lambda\left(f_{0}-\varepsilon\right) A_{1} \Sigma_{s=0}^{T-1} b(s) \frac{\alpha-|c|}{1-c^{2}}\|y\|_{X}>\|y\|_{X} .
$$

If $0<f_{\infty}<\infty$, then there exists $\tilde{H}>0$ satisfying for any $u \geq \tilde{H}, f(u) \leq\left(f_{\infty}+\varepsilon\right) u$. Take $r_{2}=\max \left\{2 r_{1},\left(\left(1-c^{2}\right) /(\alpha-|c|)\right) \tilde{H}\right\} . y \in \partial \Omega_{r_{2}}$ tells that $\left(A^{-1} y\right)(n-\tau(n)) \geq((\alpha-$ $\left.|c|) /\left(1-c^{2}\right)\right)\|y\|_{x} \geq \tilde{H}$. So $f\left(\left(A^{-1} y\right)(n-\tau(n))\right) \leq\left(f_{\infty}+\varepsilon\right)\left(A^{-1} y\right)(n-\tau(n))$ for $y \in \partial \Omega_{r_{2}}$. Thus, by Lemma 2.8 , for $y \in \partial \Omega_{r_{2}}$, we have

$$
\|Q y\|_{X} \leq \frac{\lambda B\left(f_{\infty}+\varepsilon\right) \sum_{s=0}^{T-1} b(s)+B L|c| \sum_{s=0}^{T-1} a(s)}{1-|c|}\|y\|_{X}<\|y\|_{X} .
$$

It follows from Lemma 2.1 that

$$
i\left(Q, \Omega_{r_{1}}, K\right)=0, \quad i\left(Q, \Omega_{r_{2}}, K\right)=1 .
$$

Therefore, $i\left(Q, \Omega_{r_{2}} \backslash \bar{\Omega}_{r_{1}}, K\right)=1$ and $Q$ has at least one fixed point $y(n)$ in $\Omega_{r_{2}} \backslash \bar{\Omega}_{r_{1}}$. Lemma 2.6 shows that $\left(A^{-1} y\right)(n)$ is a positive $T$-periodic solution of (1.2).

Our results are applicable to consider multiplicity of periodic solutions for many neutral difference equations.

Example 3.4. We consider the following neutral difference equation:

$$
\Delta\left[u(n)+\frac{1}{3} u(n-1)\right]=\frac{1}{4} u(n)-\lambda[1-\sin \pi n] u^{a}(n-\tau(n)) e^{-u(n-\tau(n))}, \quad n \in \mathbb{Z},
$$

where $\lambda$ and $a$ are two positive parameters, $\tau(n+2) \equiv \tau(n)$. Take $\tau=1, c=-1 / 3, a(n) \equiv$ $1 / 4, b(n)=1-\sin \pi n, g(u) \equiv 1, f(u)=u^{a} e^{-u}, L=l=1$. Then assumptions $\left(\mathrm{E}_{1}\right)$ and $\left(\mathrm{E}_{2}\right)$ hold, $f_{\infty}=0$, and $\max _{u \in[0, \infty)} f(u)=f(a)$.

By direct computations, we have $k=\alpha=2 / 5, f_{0}=+\infty$ if $a \in(0,1), f_{0}=1$ when $a=1$, and $f_{0}=0$ as $a>1$. Furthermore, let $t_{0}=\min \{a,(3 / 2)\}$, we have

$$
\begin{gathered}
M(1)=\max \left\{f(t): 0 \leq t \leq \frac{3}{2}\right\}=f\left(t_{0}\right), \\
m(1)=\min \left\{f(t): \frac{3}{40} \leq t \leq \frac{3}{2}\right\}=\min \left\{f\left(\frac{3}{2}\right), f\left(\frac{3}{40}\right)\right\}=r_{0} .
\end{gathered}
$$


Thus

$$
\lambda_{0}=\frac{1}{A_{1} m(1) \sum_{s=0}^{T-1} b(s)}=\frac{3}{4 r_{0}}, \quad \lambda_{1}=\frac{1-|c|-B L|c| \sum_{s=0}^{T-1} a(s)}{B M(1) \sum_{s=0}^{T-1} b(s)(1-|c|)}=\frac{7}{40 f\left(t_{0}\right)}
$$

Applying Theorem 3.1 to (3.24), we obtain the following results.

\section{Conclusion}

(a) If $a \in(0,1)$, then (3.24) has one positive two-periodic solution for $\lambda>3 / 4 r_{0}>0$ or $0<\lambda<7 / 40 f(a)$.

(b) If $a=1$, then (3.24) has one positive two-periodic solution for $\lambda>3 / 4 r_{0}>0$.

(c) If $a>1$, then (3.24) has two positive two-periodic solutions for $\lambda>3 / 4 r_{0}>0$.

\section{References}

[1] R. P. Agarwal and W. N. Zhang, Periodic solutions of difference equations with general periodicity, Computers \& Mathematics with Applications 42 (2001), no. 3-5, 719-727.

[2] S. N. Chow, Existence of periodic solutions of autonomous functional differential equations, Journal of Differential Equations 15 (1974), no. 2, 350-378.

[3] K. Deimling, Nonlinear Functional Analysis, Springer, Berlin, 1985.

[4] M. Fan and K. Wang, Periodic solutions of a discrete time nonautonomous ratio-dependent predator-prey system, Mathematical and Computer Modelling 35 (2002), no. 9-10, 951-961.

[5] D. J. Guo and V. Lakshmikantham, Nonlinear Problems in Abstract Cones, Notes and Reports in Mathematics in Science and Engineering, vol. 5, Academic Press, Massachusetts, 1988.

[6] D. Jiang, J. Chua, and M. Zhang, Multiplicity of positive periodic solutions to superlinear repulsive singular equations, Journal of Differential Equations 211 (2005), no. 2, 282-302.

[7] M. A. Krasnosel'skiǔ, Positive Solutions of Operators Equations, Noordhoff, Groningen, 1964.

[8] B. S. Lalli and B. G. Zhang, On existence of positive solutions and bounded oscillations for neutral difference equations, Journal of Mathematical Analysis and Applications 166 (1992), no. 1, 272287.

[9] Y. K. Li, L. F. Zhu, and P. Liu, Positive periodic solutions of nonlinear functional difference equations depending on a parameter, Computers \& Mathematics with Applications 48 (2004), no. 10-11, 1453-1459.

[10] S. Lu and W. Ge, Periodic solutions of neutral differential equation with multiple deviating arguments, Applied Mathematics and Computation 156 (2004), no. 3, 705-717.

[11] M. C. Mackey and L. Glass, Oscillations and chaos in physiological control systems, Science 197 (1997), 287-289.

[12] H. Péics, Positive solutions of neutral delay difference equation, Novi Sad Journal of Mathematics 32 (2005), no. 2, 111-122.

[13] H. L. Smith and Y. Kuang, Periodic solutions of differential delay equations with threshold-type delays, Oscillation and Dynamics in Delay Equations (San Francisco, CA, 1991), Contemporary Mathematics, vol. 129, American Mathematical Society, Rhode Island, 1992, pp. 153-176.

[14] H. Wang, Positive periodic solutions of functional differential equations, Journal of Differential Equations 202 (2004), no. 2, 354-366.

[15] M. Ważewska-Czyżewska and A. Lasota, Mathematical problems of the dynamics of a system of red blood cells, Roczniki Polskiego Towarzystwa Matematycznego. Seria III. Matematyka Stosowana 6 (1976), 23-40. 


\section{Two solutions of neutral difference equations}

[16] X. Y. Zeng, B. Shi, and M. J. Gai, A discrete periodic Lotka-Volterra system with delays, Computers \& Mathematics with Applications 47 (2004), no. 4-5, 491-500.

[17] M. R. Zhang, Periodic solutions of linear and quasilinear neutral functional-differential equations, Journal of Mathematical Analysis and Applications 189 (1995), no. 2, 378-392.

[18] G. Zhang and S. S. Cheng, Positive periodic solutions of coupled delay differential systems depending on two parameters, Taiwanese Journal of Mathematics 8 (2004), no. 4, 639-652.

Jun Wu: College of Mathematics and Computer Science, Changsha University of Science Technology, Changsha 410076, China

E-mail address: junwmath@hotmail.com

Yicheng Liu: Department of Mathematics and System Science, College of Science,

National University of Defense Technology, Changsha 410073, China

E-mail address: liuyc2001@hotmail.com 


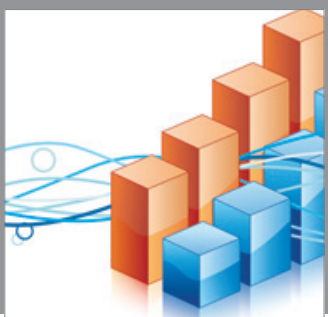

Advances in

Operations Research

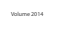

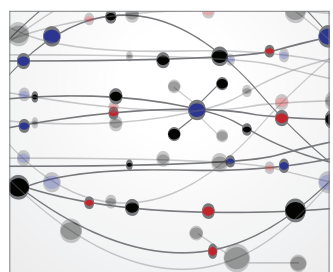

\section{The Scientific} World Journal
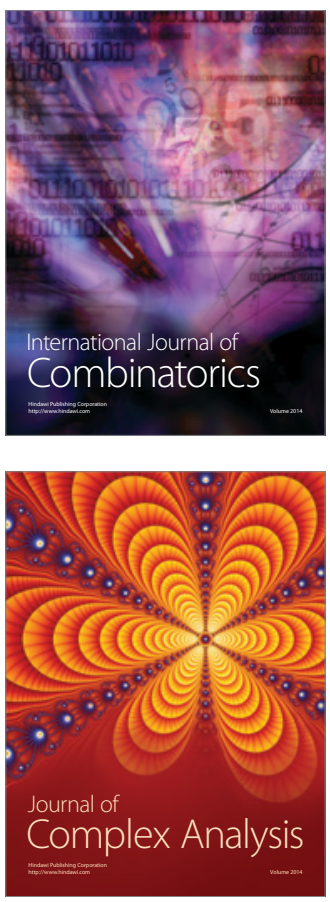

International Journal of

Mathematics and

Mathematical

Sciences
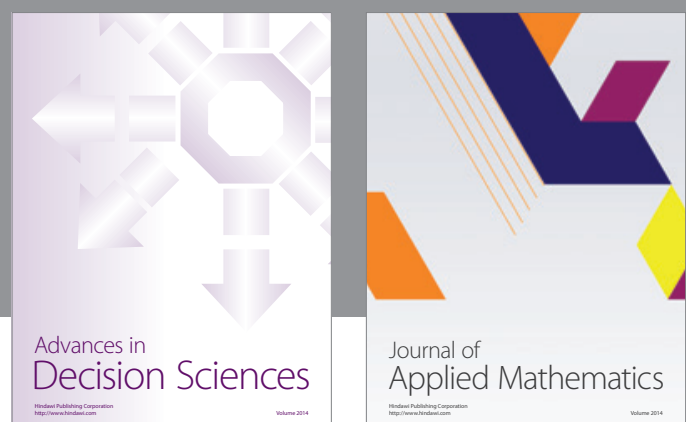

Journal of

Applied Mathematics
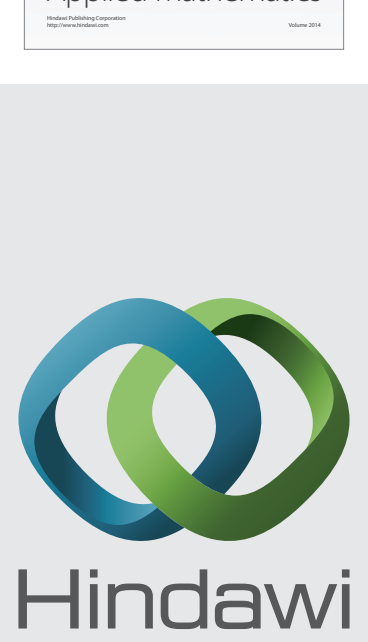

Submit your manuscripts at http://www.hindawi.com
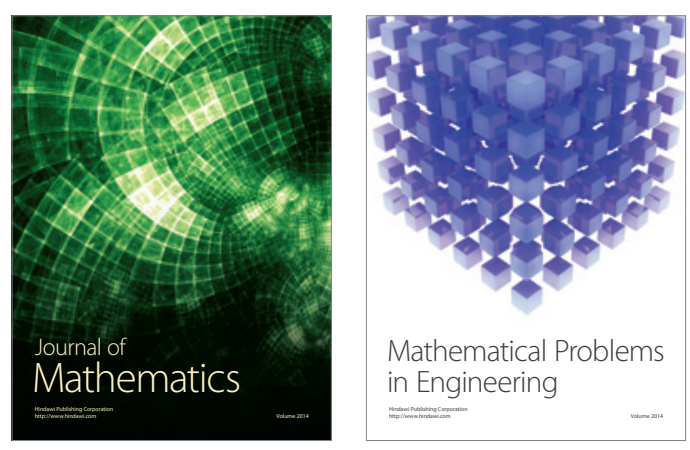

Mathematical Problems in Engineering
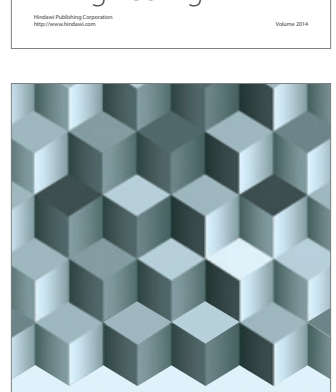

Journal of

Function Spaces
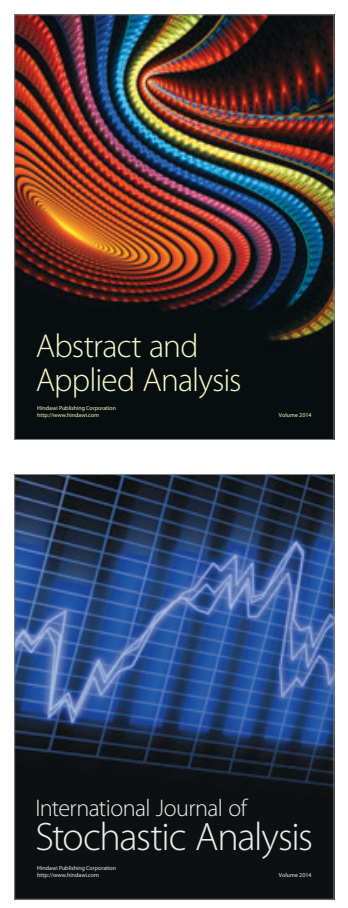

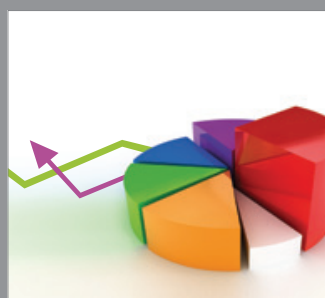

ournal of

Probability and Statistics

Promensencen
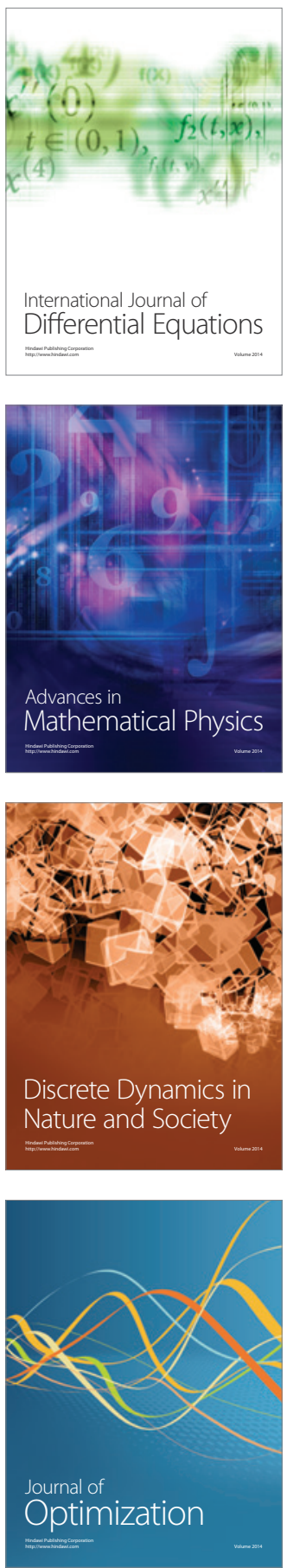